\title{
RELACIÓN ENTRE LAS ORIENTACIONES DE META Y EL CLIMA MOTIVACIONAL PERCIBIDO EN EL DEPORTE.
}

\author{
Zulma Daza ${ }^{1}$, Jairo Flórez ${ }^{2}$, Yofre Sanabria ${ }^{3}$
}

1. Mg (c). Pedagogía de la Cultura Física. InvestigadorTendencias Pedagógicas.UPTC, Tunja. zulyoli48@yahoo.es

2. PhD. Ciencias de la Actividad Física y el Deporte. Profesor Asistente. Escuela de Educación Física Recreación y Deporte. UPTC, Tunja. Grupo de investigación Tendencias Pedagógicas. UPTC Tunja. jairo.flórez01@uptc.edu.co

3. Mg Pedagogía de la Cultura Física. InvestigadorTendencias Pedagógicas.UPTC, Coordinador Académico. Maestría en Pedagogía de la Cultura Tunja.maestria.pcf@uptc.edu.co

\section{RESUMEN:}

La relación existente entre las orientaciones de meta y el clima motivacional percibido en el deporte determina unos perfiles. Un primer perfil con altas metas de logro, un segundo perfil con metas moderadas, un tercer perfil con metas bajas y un cuarto perfil caracterizado por altas metas de aproximación y maestría. Aquí se puede hacer un análisis multivalente donde se evidenciaría que los deportistas del primer y cuarto perfil percibirán un clima motivacional más orientado hacia la tarea, y los deportistas situados en el segundo y tercer perfil percibirían un clima motivacional orientado más hacia el ego.

\section{Objetivo}

Realizar una revisión teórica de los conceptos y estudios realizados sobre la relación entre el clima motivacional percibido y las orientaciones de meta en el contexto deportivo

Materiales y métodos

Este es un estudio de revisión sistemática, sustentado en las diferentes bases de datos a las cuales está inscrita la biblioteca de la Universidad Pedagógica y Tecnológica de Colombia, como: DIGITALIA, DIALNET, EBSCO HOST, ELIBRO, PROQUEST, ACADEMIC SEARCH PREMIER, entre otras.

Resultados

Se rescataron 100 artículos de los cuales se seleccionaron aquellos con criterios de publicación y que presentan indicadores de relación entre las orientaciones de meta y el clima motivacional percibido en el deporte.

Conclusiones 
El clima motivacional orientado hacia la tarea se relaciona positivamente con la orientación disposicional a la mima y negativamente con el clima motivacional al ego y la orientación al ego, mientras que el clima motivacional al ego se asocia positivamente con la orientación al ego y negativamente con el clima motivacional a la tarea. Se requiere más estudios que son necesarios para corroborar los resultados encontrados con diferentes poblaciones y culturas.

PALABRAS CLAVE: Clima motivacional percibido, Orientaciones de Meta.

\title{
RELATIONSHIP BETWEEN PERCEIVED MOTIVATIONAL CLIMATE AND GOAL ORIENTATION IN SPORT.
}

\begin{abstract}
The relationship between goal orientations and perceived sport motivational climate determines profiles in athletes. A first profile with high goals of achievement, a second profile with moderate goals, a third profile with low goals and a fourth profile characterized by high goals approach and mastery. Here, you can make a multivariate analysis where evidence of the first and fourth profile athletes would receive more motivational climate task- oriented and athletes located on the second and third profile would receive more motivational climate ego-oriented.

Objective

To conduct a theoretical review of concepts and studies on the relationship between the perceived motivational climate and the guidelines of goal in the sport context.

\section{Materials and methods}

This is a study of systematic review, based on the different databases to which the library of the Pedagogy and Technology University of Colombia, is inscribed: DIGITALIA, DIALNET, EBSCO HOST, e-book, PROQUEST, ACADEMIC SEARCH PREMIER, among others.
\end{abstract}

Results

One hundred articles were rescued of selected with the criteria of publication and presenting indicators of relationship between the goal orientations and the motivational climate seen in the sport.

\section{Conclusions}

The motivational climate to the task is related positively dispositional orientation to this and negatively motivational climate to the ego and the ego-oriented, 
while ego motivational climate is positively associated with orientation to the ego and negatively with the motivational climate to the task. It requires more studies to corroborate the results found with different populations and cultures.

Key words: Perceived motivational climate, goal orientations.

\section{INTRODUCCIÓN}

La motivación es uno de los procesos de pensamiento más estudiado, complejo y de mayor importancia dentro del proceso de enseñanza-aprendizaje; estudiado porque es innumerable la cantidad de artículos y libros que abordan esta temática, complejo porque este proceso incluye muchos aspectos que interactúan estrechamente como la personalidad, variables externas, cognitivas, biológicas y emociones), e importante porque podría explicar muchas de las cuestiones que cotidianamente encontramos en nuestras clases como por ejemplo, a algunos estudiantes les gusta las actividades físico-deportivas, a otros no, algunos se implican más activamente, otros no; entre otras.

Cuando se habla de motivación, nos estamos refiriendo a sus tres dimensiones: la dirección; las razones que llevan al individuo a escoger una determinada actividad (o a evitarla); la intensidad; el mayor o menor esfuerzo que el individuo emplea en la actividad y la duración; el tiempo que puede mantener el interés y el esfuerzo. Así, podemos decir que la motivación es aquello que inicia, mantiene y convierte en más o menos intensa la actividad de los individuos para alcanzar una meta.

Para explicar la motivación, habrá que recurrir a muchos conceptos y sus interrelaciones, ya que el exclusivismo conceptual dificultara el abordaje de la complejidad de actividades y eventos que tienen lugar en las instalaciones deportivas de los colegios e instituciones en relación con el objeto de estudio.
En los últimos 20 años, la teoría de las perspectivas de las metas de logro se ha convertido en un marco referencial en el estudio de la motivación en el deporte y la educación física (Duda 1992, Papaioannou 1995 1, Treasure y Roberts 1995). Sostiene que existen dos perspectivas de meta importantes que actúan en las actividades de logro: una implicación en la tarea y una implicación en el ego. Se relacionan con diferentes formas de definir el éxito y de juzgar el nivel de competencia personal en contextos de logros concretos. Cuando se está implicado en la tarea, las percepciones de competencia son auto referenciadas y las experiencias del dominio de la tarea, de esfuerzo, de aprendizaje y mejora personal producen un sentimiento de éxito. Cuando se está en un estado de implicación en el ego, la competencia percibida toma un punto de referencia normativo, sienten que tienen éxito cuando demuestran que son mejores que los demás.

La perspectiva de meta adoptada en una situación particular influye en cómo la gente siente, interpreta y responde a las actividades de logro. Se asume que un análisis de las diferencias entre las personas en cuanto a las metas de logro y las percepciones de competencia proporcionara una mejor comprensión de las variaciones en los patrones de motivación.

La implicación a la tarea está relacionada con cogniciones y conductas de logro positivas y adaptativas, como la persistencia, el reto, el esfuerzo, esto surge si la persona en cuestión tiene una alta 0 baja capacidad percibida. La implicación en 
el ego, a corto plazo, también producirá un patrón de logro positivo, pero siempre que la persona no tenga dudas sobre la idoneidad de su capacidad.

Donde predomina la implicación en el ego y la persona cree que no podrá demostrar una capacidad superior, es más probable que se produzcan cogniciones y conductas de logro negativas, ejecución deteriorada, abandono, falta de interés, elección de tareas demasiado fáciles o demasiado difíciles.

La probabilidad de que una persona esté implicada en la tarea 0 en el ego en situaciones de logro, como las clases de educación física o el deporte, depende tanto de las diferencias individuales como de la situación concreta. Cuando se habla de diferencias individuales se refiere a que nosotros variamos en nuestra tendencia disposicional a estar implicados a la tarea y al ego 0 al grado de orientación a la tarea y al ego. Estas orientaciones aparecen como ortogonales, a partir de los 10 años, es decir, las personas pueden tener puntuaciones altas en ambas orientaciones, alta en una y baja en otra, o baja en ambas orientaciones.

Se hace necesario determinar, dentro del campo de la motivación de logro, las orientaciones de meta y el clima motivacional percibido en los deportistas, como punto de partida para futuras intervenciones en el campo del deporte, y así, realizar las correcciones necesarias en el plano de la didáctica y la metodología utilizadas en los entrenamientos.

En Colombia son pocos los estudios realizados donde se indique un punto de partida para este tipo de investigación. A nivel mundial se ha adentrado mucho en este campo de la psicología aplicada a la actividad física y el deporte, principalmente en España, entre ellos; esta la investigación realizada por Portal y Cols, (2000); donde hace una consideración de evaluación de la motivación hacia el proceso del entrenamiento deportivo realizada en una población de deportistas universitarios.

Entre otras investigaciones que se deben tener en cuenta para la realización de esta investigación son los estudios hechos porCervelló y Santos-Rosa, (2000); Viciana y Cols, (2003); Cervelló y Jiménez, (2001); Castillo y Cols, (2000); Llanos y Tabernero, (2003); Iglesias y Cols, (2003); Pérez y Delgado, (2003); López y Márquez, (2001); Salguero y Cols, (2002); Salguero y Cols, (2003); Gonzáles y Cols, (2000); Peiró, (1999).

Si la educación física y los programas de deporte organizado busca fomentar estilos de vida activos en el estudiante y los deportistas, la creación de ambientes de clase que impliquen en la tarea y la facilitación de una orientación a la tarea, tanto en las clases como en otros contextos de la actividad física, pueden ser cruciales en la consecución de este.

Con respecto a las metas del contexto 0 situacionales, investigaciones en la educación física y el deporte, han estudiado la naturaleza y las consecuencias del clima motivacional percibido, Ames (1992), afirma que el clima motivacional se considera multidimensional y depende de factores como la estructura de las recompensas, las bases para el sistema de evaluación y la comunicación de dicha evaluación, los criterios utilizados para el agrupamiento. El clima motivacional se considera como la apreciación que el alumnado de educación física o el deportista hacen del ambiente de clase 0 sesión de entrenamiento, este ambiente puede estar orientado al ego $0 \mathrm{a}$ la tarea.

Por ejemplo; Papaioannou y Kouli (1999), en una clase de educación física de 45 minutos, programaron actividades orientadas a la tarea y al ego, en la misma sesión. Cuando los estudiantes participaban en una sesión orientada a la tarea, demostraban un mayor grado de confianza, 
menor ansiedad somática y percibía que la sesión implicaba más en la tarea que en el ego. También se encuentran diferencias disposicionales entre los estudiantes. Independientemente de que las actividades se diseñaran para implicar en la tarea o en el ego, si los estudiantes estaban muy orientados en la tarea, mostraban una mayor implicación, una pérdida de autoconciencia y más experiencias cumbre.

Hay que tener en cuenta el trato diferencial y potencial que reciben los estudiantes con una alta y una baja capacidad en ambientes que están más orientados a la tarea o que implican más en el ego. Cuando el ambiente de la educación física estaba muy orientado a la tarea, los estudiantes percibían un trato menos diferencial hacia los más y menos capaces.

Papaioannou (1995), también encontró en el contexto deportivo, cuyo ambiente percibido implicaba en la tarea, el deportista vio menos diferencias en el trato a los chicos y las chicas por parte del entrenador, tanto en el enfoque del aprendizaje, el refuerzo, la autonomía como en el ajuste de la sesión de entrenamiento.

Cuando los ambientes de educación física y el deporte están orientados al ego, el estudiante con baja percepción de capacidad corre el riesgo de ver disminuido su sentido de competencia. Esta disminución en las percepciones de competencia tiene implicaciones para la participación diaria del estudiante "de riesgo" en la E.F y el deporte y disminuye la probabilidad de que adopten un estilo de vida activo hasta llegar a la edad adulta.

\section{MATERIALES Y MÉTODOS}

Revisión de las diferentes bases de datos a las cuales está inscrita la biblioteca de la Universidad Pedagógica y Tecnológica de Colombia, como: DIGITALIA, DIALNET, EBSCO HOST, E-LIBRO, PROQUEST,
ACADEMIC SEARCH PREMIER, entre otras.

\section{Instrumento}

Los cuestionarios que se van a tener en cuenta para recolectar la información desde su revisión sistemática son:

- Cuestionario para valorar las Orientaciones de Meta. Adaptado a la educación física por Walling y Duda (1995), TEOSQ; validado por Peiró y cols. (2004). Consta de 16 items, midiendo la orientación a la tarea (8 ítems) y la orientación al ego (8 ítems).

- Cuestionario de Percepción del Clima Motivacional en las Clases de Educación Física (Cervelló y cols., 2002b). PMCSQ-2. Consta de 24 ítems y posee dos dimensiones: Percepción del Clima Motivacional Orientado a la tarea; y Percepción del Clima Motivacional orientado al ego.

\section{RESULTADOS}

\begin{tabular}{|c|c|c|c|c|c|}
\hline $\begin{array}{c}\text { Nombro del } \\
\text { arficulo }\end{array}$ & Autor|(s) & Resumen & Revista & Año & Musetra \\
\hline 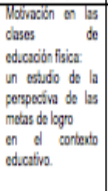 & \begin{tabular}{|l|} 
Esuarbo II \\
Convelo, \\
Francisco \\
Sartso Rosa
\end{tabular} &  & 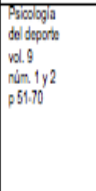 & 2000 & $\begin{array}{l}290 \text { estudiantiss } \\
153 \text { hombres, } 127 \\
\text { myjers } \\
14 \text { y } 18 \text { anios }\end{array}$ \\
\hline 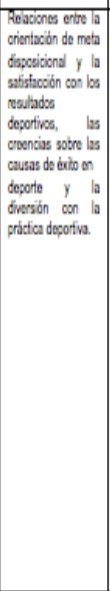 & 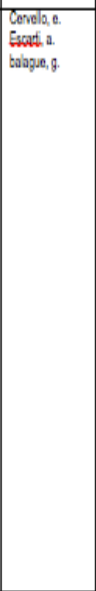 & 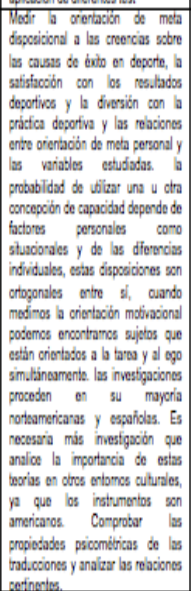 & 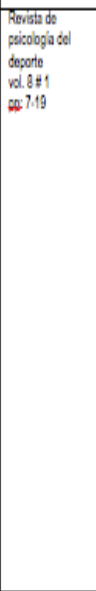 & 7987 & 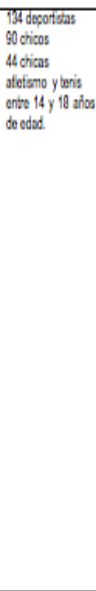 \\
\hline
\end{tabular}



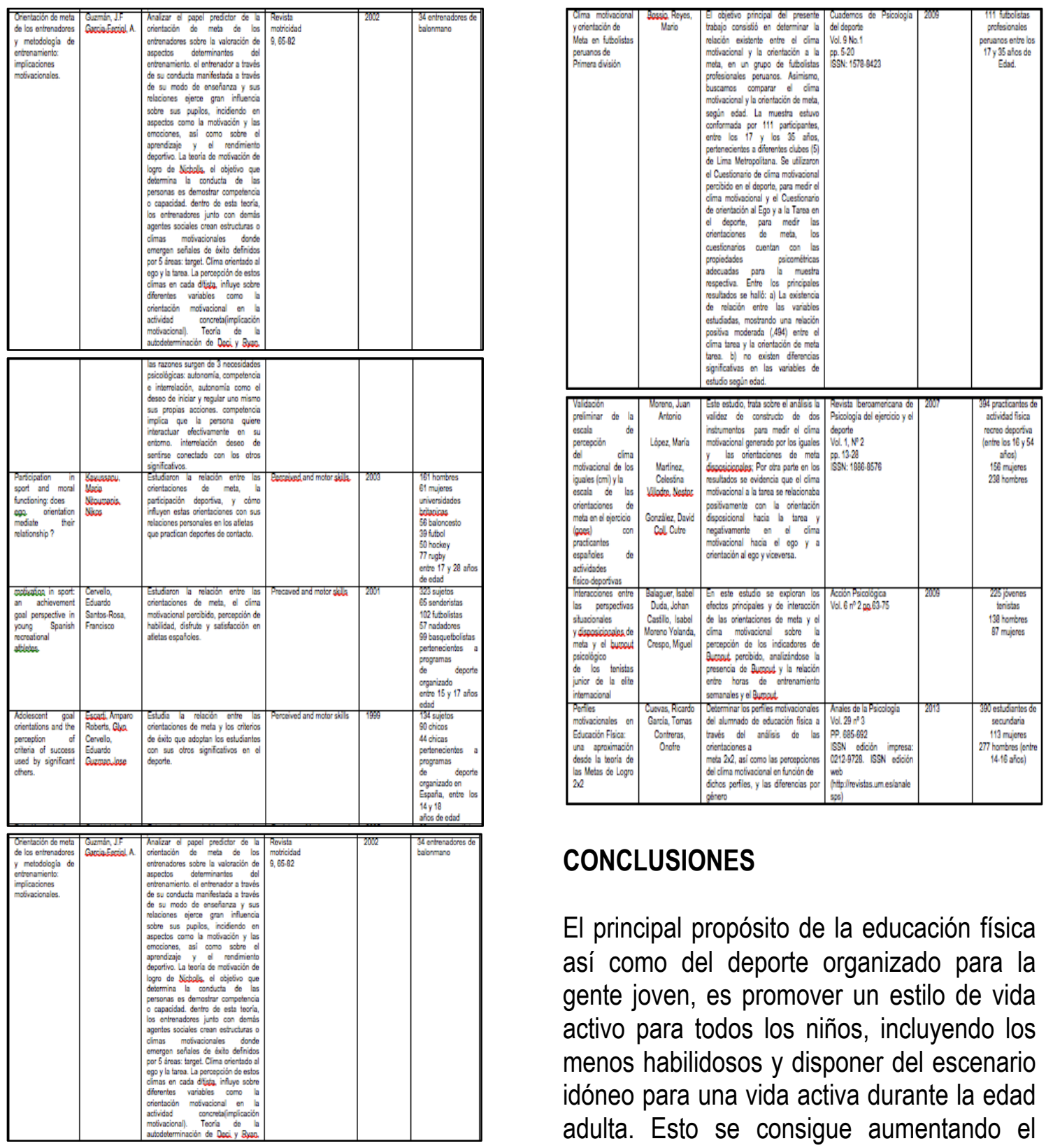

\section{CONCLUSIONES}

El principal propósito de la educación física así como del deporte organizado para la gente joven, es promover un estilo de vida activo para todos los niños, incluyendo los menos habilidosos y disponer del escenario idóneo para una vida activa durante la edad adulta. Esto se consigue aumentando el actual estado de salud de los niños y adolescentes, sus percepciones de competencia física contribuyendo a inyectar la creencia de que la actividad física es crucial para la calidad de vida. Siempre y cuando se refuerce una orientación hacia la tarea en los estudiantes de todos los niveles y un clima en la educación física y en el deporte que implique a los estudiantes y deportistas en la tarea.

La percepción de la habilidad se ha mostrado como buen predictor, (variables)la labor del profesor o entrenador presenta criterios de éxito a sus estudiantes y deportistas y no parece presentar niveles de 
orientación al ego o implicación activa de los estudiantes 0 deportistas. El clima motivacional se relaciona con la satisfacción en clase y la diversión a partir de la habilidad auto referencial. La evaluación basada en la mejora personal y la valoración depende del profesor. El ego está alejado de objetivos educativos.

La diversión y el aburrimiento más que factores independientes cabe considerarlos como extremos de una misma dimensión. La orientación a la tarea se asocia a un patrón más adaptativo que el de la orientación al ego. El ego, utiliza como fuente de información comparaciones externas, no consideran la diversión como un elemento importante. Al aparecer los primeros fracasos empiezan a declinar en su motivación. Se considera que el esfuerzo es una de las causas de éxito en el deporte y no que la utilización de trampas sea importante para conseguir el éxito. Los profesores debemos intentar implicar a deportistas y estudiantes en criterios de análisis del fracaso y éxito deportivo.

Para estudiar las implicaciones motivacionales de los resultados desde la teoría de las metas de logro se analizaran los ítems por cada orientación motivacional; ego y tarea, del entrenador y cómo se relaciona con el TARGET. Existen contradicciones con respecto a las hipótesis de Ames y los resultados de estudio. Ames dice que un clima orientado al ego y la ausencia de variabilidad de actividades es característico, pero en el estudio dice lo contrario. Lo mismo sucede con la agrupación de jugadores en función de su habilidad. Lo mismo con la especificidad del entrenamiento. Se está de acuerdo en el control de los progresos del aprendizaje sin tener en cuenta los resultados cuando se orienta a la tarea. La dirección poco autoritaria que permite la autonomía delos deportistas orientados al ego no permite a los deportistas tomar decisiones. Esto en cuanto a la teoría de motivación de logro. En cuanto a la teoría de la autodeterminación la orientación a la tarea, el entrenador otorga importancia a aspectos metodológicos que ayudan a adquirir mayor percepción de competencia con el tiempo. La interrelación con padres y familiares concede más información para interaccionar positivamente con el jugador. $Y$ la autonomía para favorecer la motivación intrínseca del deportista y mantenerlo en la práctica.

\section{REFERENCIAS:}

BALAGUER, I. CASTILLO, I. DUDA, J. GARCIA, M. Asociaciones entre la percepcion del clima motivacional creado por el entrenador, orientaciones disposicionales de meta, regulaciones motivacionales y vitalidad subjetiva en jovenes jugadores de tenis. Revista Psicologia del Deporte.2011. Vol $20 n^{0} 1 \mathrm{pp}$ 133-148

Cecchini, J.Gonzalez, C. Lopez, J. Brustad, R. RELACION DEL CLIMA MOTIVACIONAL PERCIBIDO CON LA ORIENTACION DE META, LA MOTIVACION INTRINSECA $Y$ LAS OPINIONES $Y$ CONDUCTAS DE FAIR PLAY. Revista Mexicana de Psicologia. 2005. Vol $22 n^{0} 2$ pp 469-479

Lopez, J. Balaguer, I. Castillo, I. Tristan, J. Clima motivacional percibido, motivacion autodeterminada y autoestima en jovenes deportistas mexicanos. Revista Psicologia del deporte. 2011 Vol 20 no 1 pp 209-222

Reyes, M. CLIMA MOTIVACIONAL Y ORIENTACION DE META EN FUTBOLISTAS PERUANOS DE PRIMERA DIVISION. Cuadernos de psicologia del deporte.2009.VOL 9 No 1 PP 5-20

Moreno, J. Lòpez, M. Martinez, C. Villodre, N. Gonzalez, D. Coll, C. VALIDACIÓN PRELIMINAR DE LA ESCALA DE PERCEPCION DEL CLIMA MOTIVACIONAL DE LOS IGUALES (CMI) $Y$ LA ESCALA DE LAS ORIENTACIONES 
DE META EN EL EJERCICIO (GOES) CON PRACTICANTES ESPAÑOLES DE ACTIVIDAD FISICO-DEPORTIVA. Revista de iberoamerica de psicologia del ejercicio y el deporte. Vol $1 \mathrm{n}^{0} 2 \mathrm{pp} 13-28$

Balaguer, I. Duda, I. Castillo, I. Moreno, Y. Crespo, M. INTERACCIONES ENTRE LAS PERSPECTIVAS SITUACIONALES $Y$ DISPOSICIONALES DE META Y EL BURNOUT PSICOLOGICO DE LOS TENISTAS JUNIOR DE LA ELITE INTERNACIONAL. Revista Acción psicológica. 2009 Vol 6 n² 2 pp 63-75

Cuevas, R. Garcia, T. Contreras, O. Perfiles motivacionales en educacion fisica: una aproximacion desde la teoria de las metas de logro $2 \times 2$. Revista anales de psicologia. 2013 Vol 29 n $^{0} 3$ pp 685-692

Eduardo, M. Cervello, E. Santos, F, Motivacion en las clases de educacion fisica: un estudio de las perspectivas de las metas de logro en el contexto educativo. Revista Psicologia del deporte. 200 Vol 9 n01 y 2 pp $51-70$

Cervello, E. Escarti, A. Balaguer, G. Relaciones entre las orientacion de meta disposicional y la satisfaccion con los resultados deportivos, las creencias sobre las causas de éxito en deporte y la diversion con la practica deportiva. Revista de Psicologia del deporte. 1999. Vol $8 \mathrm{n}^{0} 1 \mathrm{pp}$ 7-19

Guzman, J, Garcia, S. Ferriol, A. ORIENTACION DE META DE LOS ENTRENADORES Y METODOLOGIA DE ENTRENAMIENTO IMPLICACIONES MOTIVACIONALES, Revista

Motricidad.2002. Vol 9 pp 65 - 82

Kayussanu, M. Nitoumanis, Nitoumanis, N. Participation in sport and moral functioning: does ego orientation mediate their relationship. PERCEIVED AN MOTOR SKILLS. 2003
Cervello, E. Santos, F. MOTIVATION IN SPORT: AN ACHIEVEMENT GOAL PERSPECTIVE IN YOUNG SPANISH RECREATIONAL ATHLETES. PERCEIVED AND MOTOR SKILLS. 2001.

Escarti, A. Roberts, G. Cervello, E. Guzman, $J$. Adolescent goal orientations and the perception of criteria of succes used by significant others PERCEIVED AND MOTOR SKILLS. 1999. 